\title{
Notes Helminthologiques III: Strigeidae Railliet, Diplostomidae Poirier et Brauninidae Bosma (Trematoda)
}

par

\author{
Georges DUBOIS
}

Les observations publiées dans cette troisième série de notes ont été faites sur des matériels recueillis ou communiqués par plusieurs correspondants que nous remercions ici: le $\mathrm{D}^{\mathrm{r}}$ Robert L. Rausch, chef de la Zoonotic Disease Section, Arctic Health Research Center, à College (Alaska); le Dr Franklin SogandaresBernal, du Department of Microbiology, University of Montana, à Missoula, Mont.; le Dr John M. Kinsella, du Department of Veterinary Science, University of Florida, à Gainesville; M ${ }^{\text {lle }}$ Elisabeth van den Broek, actuellement biologiste au Zoölogisch Laboratorium der Universiteit d'Amsterdam; le Dr Simon Gretillat, directeur de l'Ecole des Assistants d'Elevage et des Agents techniques, à Niamey (République du Niger).

\section{Apharyngostrigea ibis Azim, 1935}

Le $\mathrm{D}^{\mathrm{r}}$ Simon Gretillat, de Niamey, nous a remis un matériel récolté le 5 juin 1971 dans l'intestin d'une Ardea goliath Cretzsch. Lui-même a abattu et autopsié cet oiseau à Maradi (République du Niger), localité située à $700 \mathrm{~km}$ à l'E de Niamey et à $200 \mathrm{~km}$ au $\mathrm{N}$ de Kano (Nigeria). Il s'agit, selon nous, d'Apharyngostrigea ibis Azim, qui a été retrouvée au Ghana par UKolı (1967).

L'espèce est de taille médiocre, 2,75 à $4,2 \mathrm{~mm}$, à corps généralement fortement recourbé, surtout à l'état contracté. L'un des exemplaires montre nettement l'habitus de l'organe tribocytique éversé: la lèvre dorsale se termine par deux digitations corniformes, tandis que la lèvre ventrale s'épanche en un rebord semi- 
circulaire. La glande protéolytique lobulée, ovoïde ou cucumiforme, mesure $350 / 200 \mu$ sur un spécimen en extension; son lobule postérieur est plus grand que les autres. L'ovaire est réniforme. Les tesicules sont grossièrement lobés. Les œufs, rares, mesurent 85-95/55-60 $\mu$.

\section{Ophiosoma crassicolle Dubois et Rausch, 1948}

3 spécimens faisant partie de la collection H. J. Van Cleave nous ont été remis par le D ${ }^{r}$ Robert L. Rausch. Les préparations étiquetées « Botaurus lentiginosus, Maple River, Mich., VC 1513-14 » sont incorporées à la collection de l'Institut de zoologie, Université de Neuchâtel (G.D.), nos H 71-73 (lectotype: n 71).

\section{Strigea elegans Chandler et Rausch, 1947}

Un exemplaire, long de 2,5 mm, fortement arqué, a été recueilli par Robert L. Rausch en Alaska, près de Fairbanks, le 3 septembre 1968, chez une Chouette épervière américaine, Surnia ulula caparoch (Müller) [nº 35891, ô de 154 g], qui fut capturée comme jeune oiseau emplumé et mourut en captivité au bout de quelques semaines.

Segment antérieur finement spinescent dans sa première moitié, surtout en avant et dorsalement; segment postérieur inerme. Comme l'a remarqué PEARSON (1959), le pharynx qui est contigu à la ventouse buccale, est presque aussi gros qu'elle (VB 130/115 $\mu$, PH 110/107 $\mu$ ). Le cône génital est petit (diamètre $130 \mu$ ), faiblement délimité du parenchyme. Les vitellogènes se terminent à son approche, en remontant latéralement, sans pénétrer dans les parois de la bourse copulatrice (profonde de $260 \mu$ ). Le pore excréteur, ventral, se situe à peine en arrière de la hauteur du bord postérieur de l'anneau musculaire bursal («Ringnapf») (cf. PeARSON, op. cit., p. 159).

\section{Strigea gruis Dubois et Rausch, 1964}

Le Dr John M. Kinsella, de l'University of Florida, nous a demandé de confirmer sa détermination d'un Strigéide de Grus canadensis (L.), recueilli par D. J. Forrester à Avon Park, Highlands Co., Fla., le 16 septembre 1971. Il s'agit de Strigea gruis Dub. et Rsch. Les trois spécimens que nous avons eus à l'examen présentent toutes les caractéristiques de l'espèce, dont l'aire géographique s'étend donc de l'Alaska jusqu'en Floride.

Apatemon (Apatemon) gracilis (Rudolphi, 1819)

Un exemplaire a été recueilli dans l'iléon d'un Mergus merganser L. tué le 24 janvier 1968, à Lelystad, Flevopolder (Pays-Bas). Il nous a été communiqué par M ${ }^{11}$ E. van den Broek. 
Apatemon (Australapatemon) burti (Miller, 1923)

Plusieurs lots de ce parasite [nos 37310, 37311, 37312 et 37313] ont été récoltés par Robert L. Rausch chez des Oidemia nigra americana Swainson ( $\hat{0}$, en plumage immature), le 8 juilleț 1969, capturées près de la confluence des rivières Pilgrim et Kuzatrin, au N de Nome (Seward Peninsula, Alaska). Sur plusieurs exemplaires (dont la coloration au carmin, après fixation au formol, était déficiente), on pouvait distinguer nettement le plissement du canal hermaphrodite dans un cône génital relativement gros.

\section{Apatemon (Australapatemon) minor Yamaguti, 1933}

Ce Ver a été retrouvé aux Pays-Bas, près d'Amsterdam, dans l'intestin d'Anas platyrhynchos L. (28. VI. 1960), par M. Borgsteede, étudiant au laboratoire du professeur Davids, de l'Université de cette ville (matériel transmis par $\mathrm{M}^{1 \mathrm{ll}} \mathrm{E}$. van den Broek). Malgré le médiocre état de conservation, on peut distinguer le canal hermaphrodite plissé qui traverse le petit cône génital bien délimité du parenchyme. Le pharynx, faiblement musculeux $(65 / 50 \mu)$, ne dépasse pas la demilongueur de la ventouse buccale $(130 / 125 \mu)$.

Cotylurus (Cotylurus) brevis Dubois et Rausch, 1950

Nous avons identifié avec Cotylurus brevis les exemplaires recueillis dans le rectum d'un Anas platyrhynchos L. tué en 1969 dans le Naardermeer, près d'Amster-

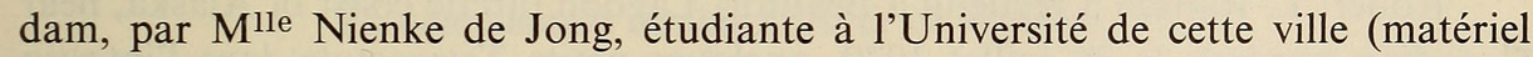
transmis par $\mathrm{M}^{\text {lle }}$ E. van den Broek).

\section{Cotylurus (Cotylurus) raabei (Bezubik, 1958)}

Ce parasite du cloaque des Canards a été retrouvé aux Pays-Bas, près d'Amsterdam, par M. Borgsteede (matériel transmis par M ${ }^{\text {lle }}$ E. van den Broek). L'hôte, Anas platyrhynchos L., est à signaler comme nouveau.

Les testicules sont lobés, à lobes lobulés et dirigés postérieurement. Les œufs sont grands $(125-136 / 73-78 \mu)$.

\section{Cotylurus (Cotylurus) strigeoides Dubois, 1958}

4 exemplaires furent recueillis par Robert L. Rausch dans l'intestin d'un Anas americana $\mathrm{Gm}$. [n $\mathrm{n}^{\circ} 37309$, ô d'environ $150 \mathrm{~g}$ ], le 8 juillet 1969 , capturé près de la confluence des rivières Pilgrim et Kuzatrin (Seward Peninsula, Alaska). 
Les Vers mesurent 1,12 à $1,16 \mathrm{~mm}$. Ils contiennent 20 à 25 œufs (94-99/62$65 \mu$ ). La ventouse ventrale n'est pas beaucoup plus grande que la buccale (comme dans le matériel original).

3 autres spécimens (dont 2 ovigères), également récoltés par Robert L. Rausch en Alaska (Beaufort Lagoon), le 20 juillet 1970, proviennent d'une Clangula hyemalis (L.) [no 38467]. Ils mesurent 0,95 à 1,20 mm. (L'exemplaire immature n'a que $0,75 \mathrm{~mm}$.) Sur le plus petit des spécimens ovigères, les 5 œufs sont relativement grands: celui qui est non déformé atteint $115 / 72 \mu$. Sur l'autre spécimen, ils ont des dimensions voisines de $100 / 65 \mu$.

Plus d'une trentaine d'exemplaires de Cotylurus strigeoides ont été récoltés par P. Duiven dans l'intestin d'un Fuligule morillon, Aythya fuligula (L.), à La Haye (Pays-Bas), le 12 septembre 1967. M1le E. van den Broek nous a communiqué ce lot pour identification. La plupart des Vers étaient mal conservés.

Lg. 1,62-1,74 mm ${ }^{1}$. SA 0,52-0,74/0,52-0,68 mm; SP 0,89-1,12/0,58-0,64 mm. Rapport des 1 . : $\mathrm{SP} / \mathrm{SA}=1,35-1,93$.

Diamètres: VB 135-150/110-150 $\mu$, PH 100/73 $\mu$, VV 175-195/157-190 $\mu$, OV 115-140/150-190 $\mu$, TA 220-400/310-420 $\mu$, TP 290-330/290-370 $\mu$, BG 90-117/125$150 \mu$. Eufs $89-102 / 57-63 \mu$. (moy. 93/61 $\mu$ ), au nombre d'une vingtaine à plus d'une quarantaine.

Situation de l'ovaire dans le SP: 8-14/100.

C'est la première mention de ce Ver en Europe.

\section{Tetracotyle sp.}

Robert L. Rausch a recueilli à Beaufort Lagoon (arctic coast of Alaska, near Canadian border), le 26 juillet 1970, quelques petits Tetracotyle dans l'intestin grêle d'un Limnodromus scolopaceus (Say) [no 38509, ô].

Il s'agit sans doute d'une larve de Cotylurus, mais son attribution spécifique reste incertaine. D'après l'hôte, elle devrait être rapportée à Cotylurus (C.) cornutus (Rudolphi, 1808), qui a été signalé par DuBois et RAUSCH (1950) au Canada, dans le même oiseau, et, par les mêmes auteurs (1960) en Alaska, chez Turdus migratorius L. D'après le lieu et la date de trouvaille, ce Tetracotyle pourrait appartenir à Cotylurus (C.) strgeoides Dubois (vide supra, no 38467).

\section{Cotylurus (Ichthyocotylurus) erraticus (Rudolphi, 1809)}

Une quarantaine d'exemplaires ont été récoltés par Robert L. Rausch, dans l'intestin d'un Larus canus brachyrhynchus Richardson [no 37322], le 8 juillet 1969, près de New Igloo, Kuzatrin River (Alaska).

1 Voir les abréviations dans le «Synopsis des Strigeidae et des Diplostomatidae (Trematoda) », p. 13 (DuBors, 1968). 
Ces exemplaires - la plupart encore jeunes mais ovigères - mesurent 1,4 à 2,4 mm. Par leur segment antérieur, en particulier, ils ressemblent à ceux que nous avons signalés (1967, pp. 698-699) ches Larus argentatus Pont., au lac Minchumina.

C. Swennen, du Nederlands Instituut voor Onderzoek der Zee, à Texel (PaysBas), a obtenu, chez Larus argentatus, de beaux exemplaires (déterminés par nous) de C. erraticus, à partir de métacercaires hébergées par Osmerus eperlanus L. ${ }^{1}$ (février-mars 1970, nos 106, 107 et 109). Cet auteur avait déjà recueilli $C$. erraticus à Texel (décembre 1969, no 94) dans le Goéland argenté (infestation naturelle).

\section{Cotylurus (Ichthyocotylurus) pileatus (Rudolphi, 1802)}

Jilji SitKo (1968, p. 289, fig. 6a) a décrit un spécimen de Cotylurus pileatus (Rud.), du gros intestin d'un Larus ridibundus L., en Tchécoslovaquie. D'après cette description, les ventouses sont «well developed »: VB 111/130 $\mu$, VV 261/193 $\mu$ (pour un Ver mesurant 2,26 mm). Le rapport: largeur SA / $\varnothing$ moyen $\mathrm{VV}=3,7$.

Les matériaux du Musée de Berlin (no 1394: coll. Rudolphi, de "Sterna cantiaca »; no 3097: coll. Wagener, de "Sterna nigra ») donnent pour ce rapport les valeurs 4,2 à 4,5 et 3,5 respectivement. Ces dernières constituent une caractéristique de l'espèce, par laquelle elle se distingue de Cotylurus platycephalus (Creplin), dont les valeurs du même rapport varient entre 6,4 et 10 .

Cotylurus pileatus présente donc in toto des ventouses bien développées et toujours apparentes (DUBOIs, 1938, p. 137; 1968, p. 220).

\section{Pseudapatemon eroliae (Fisher et Webster, 1954)}

Cette espèce, décrite d'après un abondant matériel récolté en 1942, au Texas, chez un seul Bécasseau, a été retrouvée par Robert L. Rausch en Alaska, à Point Woronzof (Anchorage), le 31 mai 1967, chez un Limnodromus griseus (Gm.) [no 34959].

Les Vers mesurent 1,56 à 2,17 mm. L'organe tribocytique massif, en forme de bouchon implanté dans le segment antérieur cupuliforme, est envahi par les follicules vitellogènes qui peuvent s'y condenser bilatéralement. (Ceux-ci ne pénètrent jamais dans les parois du segment, - d'où l'appartenance aux Cotylurini.)

La ventouse ventrale est relativement grande (170-190/180-200 $\mu)$, la ventouse buccale plus petite $(120-142 / 127-150 \mu)$. Le pharynx n'atteint que $73-80 / 65-73 \mu$. Les œufs, au nombre de 5 à 45 , mesurent 89-98/60-68 $\mu$.

${ }^{1}$ Le taux d'infestation de l'Eperlan dans l'Ijsselmeer est de 100\%. Les métacercaires, un peu plus petites que celles de Cotylurus platycephalus (Crepl.), ne sont localisées que dans le péricarde (communication de C. Swennen, in litteris, 16 mars 1971). 
Diplostomum (Diplostomum) gavium (Guberlet, 1922)

6 exemplaires ont été récoltés par Robert L. Rausch en Alaska, dans un Plongeon à bec jaune, Gavia adamsi (Gray). Aucune autre indication d'origine.

\section{Diplostomum (Diplostomum) mergi mergi Dubois, 1932}

Plus d'une centaine d'exemplaires ont été récoltés dans l'iléon de 6 Mergus merganser L. tués le 24 janvier 1968 à Lelystad, Flevopolder (Pays-Bas). Ils nous ont été communiqués par $\mathrm{M}^{11 \mathrm{e}} \mathrm{E}$. van den Broek.

Description. - SA spatuliforme, de contour longuement piriforme à lyriforme en extension, à largeur maximum au niveau de l'OT, à bords latéraux étroitement repliés ventralement, à extrémité céphalique trilobée, à PV chromophiles, réniformes ou virguliformes, parfois en croissant, sous-tendant les lobes latéraux et délimitant le lobe médian plus grand, occupé par la VB et le $\mathrm{PH}$, à glandes prosdétiques localisées dans la première moitié du segment. SP ovoïde, nettement délimité du précédent, toujours plus court et plus étroit. VV à peine en arrière de la mi-longueur du SA, plus grande que la VB; PH étroit; OT circulaire et fongiforme en expansion, ovale en rétraction, s'ouvrant par une fente médiane. OV ovoïde, submédian ou sublatéral, situé tout au début du SP; TA asymétrique, cunéiforme; TP bilobé, excavé ventro-postérieurement. VG s'avançant jusqu'au niveau de la VV ou plus en avant, constituant en arrière des TT deux amas de follicules latéro-terminaux le plus souvent assez denses; RV et GM intertesticulaires, celle-ci latérale.

Lg. $1,25-1,69 \mathrm{~mm}$. SA $0,78-1,11 / 0,33-0,47 \mathrm{~mm}$; SP 0,47-0,73/0,28-0,36 mm. Rapport des 1g.: SP/SA $=0,55-0,76$ (moy. 0,62).

Diamètres: VB 55-73/60-73 $\mu$, PH 47-65/32-39 $\mu$, VV 68-78/78-94 $\mu$, OT 150230/135-190 $\mu$, OV 63-100/92-115 $\mu$, TA 135-180/170-250 $\mu$, TP 130-230/250-330 $\mu$. Eufs $94-110 / 60-70 \mu$ (moy. 101/65 $\mu$ ), au nombre de 1 à 8 .

Longueurs: PV 75-120 $\mu$, OE 40-50 $\mu$,

Distances: du bord postérieur de la VV au bord antérieur de l'OT: 60-115 $\mu$; du rebord dorsal de la $\mathrm{BC}$ à l'extrémité du corps: $60-95 \mu$.

Situation dans le SA: limite des VG 37-57/100, VV 50-57/100 (moy. 55/100), bord frontal de l'OT 65-84/100.

Situation dans le SP: OV 2-14/100, bord frontal du TA 5-18/100, bord caudal du TP 58-68/100, rebord dorsal de la BC 85-90/100.

1 Voir les abréviations dans le «Synopsis des Strigeidae et des Diplostomatidae (Trematoda)», p. 259 (DuBoIs, 1968). 
Diplostomum (Diplostomum) spathaceum huronense (La Rue, 1927)

Ce parasite de Laridés a été retrouvé par Robert L. Rausch en Alaska, près d'Anchorage, chez deux Larus glaucescens Naumann, le 17 juillet 1951, à Cook Inlet [no 9397], et le 26 juillet 1956, à Susitna Flats [nº 17422].

La sous-espèce est bien caractérisée par le grand développement des testicules.

\section{Diplostomum (Tylodelphys) podicipinum robrauschi Dubois, 1969}

En provoquant la digestion artificielle de poissons, en Alaska, Robert L. Rausch a libéré de très jeunes larves diplostomiennes, linguiformes, longuement elliptiques, à segment postérieur conique, non ou à peine différencié, qui infestaient Dallia pectoralis Bean (Emmonak, near mouth of Yukan River, 30 mars 1970) et Cottus cognatus Richardson (Upper Kenai Peninsula, 26 mai 1970). Ces larves semblent pouvoir être attribuées à Diplostomum (Tylodelphys) podicipinum robrauschi Dub., si fréquemment trouvé en Alaska, et plus particulièrement dans l'Upper Kenai Peninsula (cf. DuBoIs, 1969, pp. 13-16).

Les mesures prises sur 10 exemplaires provenant de Cottus sont les suivantes: longueur 1,05-1,26 mm (moy. 1,19 mm); largeur 0,32-0,37 mm (moy. 0,34 mm); ventouse buccale $58-65 / 40-47 \mu$ (moy. $62 / 43 \mu$ ); ventouse ventrale plus grande et plus musculeuse, 63-78/57-65 $\mu$ (moy. 71/61 $\mu$ ); organe tribocytique oblong, s'ouvrant par une fente médiane, 165-200/70-82 $\mu$ (moy. 183/75 $\mu$ ). Les pseudo-ventouses ne sont pas encore différenciées. Le pharynx est petit, étroit; les ébauches génitales sont très rudimentaires, agglomérées.

Ces métacercaires ressemblent beaucoup à celles de "Tylodelphys podicipina » (cf. NiEWIADOMSKA, 1970, fig. 5a), notamment en ce qui concerne les proportions des ventouses, la forme de l'organe tribocytique, celle de la vésicule excrétrice, l'aspect rudimentaire et la situation des gonades.

Les larves provenant de Dallia sont moins développées: elles n'atteignent que $0,60-0,72 / 0,31-0,36 \mathrm{~mm}$. Chez les plus grandes, le segment postérieur est à peine différencié.

\section{Neodiplostomum (Conodiplostomum) spathula banghami Penrod, 1947}

10 exemplaires de ce parasite nord-américain de Falconiformes ont été recueillis par Robert L. Rausch en Alaska (Napaktualuitch Mt., « just north of Anaktuvuk Pass, Brooks Range »), le 20 avril 1971, dans un Aquila chrysaetos (L.) [no 39438].

Ces Vers mesurent 1,85 à $2,17 \mathrm{~mm}$. Les vitellogènes, très développés dans le segment antérieur, s'avancent jusqu'à une faible distance de la bifurcation intestinale ou atteignent même celle-ci. La ventouse ventrale se situe au $1 / 3$ ou aux $2 / 5$ 
de la longueur de ce segment. L'organe tribocytique est longuement elliptique. L'ovaire réniforme est très grand; il se trouve tout au début du segment postérieur.

\section{Neodiplostomum (Neodiplostomum) spathoides Dubois, 1937}

C'est à cette espèce qu'il faut rapporter le Neodiplostomum perlatum que Jilji SiтKo (1969, p. 86 et fig. 8) a décrit comme parasite de Falco cherrug Gray, en Tchécoslovaquie (Strachotin).

C'est également à elle que nous croyons pouvoir attribuer le spécimen que Odening (1970, p. 173, 176, fig. 5f) obtint d'un Asio flammeus (Pont.) et qu'il considéra comme un "fragliches Exemplar» de Neodiplostomum ( $N$.) morenodathei Oden. L'hôte, importé du Canada le 28 juillet 1963, a été autopsié le 30 octobre: il a dû probablement s'infester durant sa captivité au Zoo de Berlin.

\section{Posthodiplostomum minimum (MacCallum, 1921)}

Quelques exemplaires furent recueillis par Scott Gardner, à Gaston, Oregon, le 4 octobre 1968, dans l'intestin d'un Butorides virescens anthonyi (Mearns) [no 37268 de la collection Rausch].

\section{Alaria (Paralaria) taxideae Swanson et Erickson, 1946}

Ce parasite de Mustélidés, décrit du Minnesota, signalé ensuite au Michigan, au Maryland et en Louisiane, puis récemment au North Dakota (DYER, 1970), a été retrouvé par Robert L. Rausch chez une Hermine, Mustela erminea L., tuée à fin novembre 1971, à Galena, Alaska [n 39806, ㅇ de 246 g].

Les Vers, au nombre de 236, étaient fortement attachés à la muqueuse du duodénum, à partir d'une distance de $15 \mathrm{~mm}$ du pylore, et concentrés sur les $3 \mathrm{~cm}$ suivants. Leur accumulation provoquait un élargissement très apparent de cette partie de l'intestin.

Les 71 exemplaires que nous avons examinés ont 2 à $2,7 \mathrm{~mm}$ de longueur, 1,1 à $1,6 \mathrm{~mm}$ de largeur au niveau du segment antérieur spathacé. Le pharynx mesure 200-230/165-200 $\mu$; il est plus grand et beaucoup plus musculeux que la ventouse buccale $(130-160 / 170-210 \mu)$. La ventouse ventrale est petite $(104-115 / 115-140 \mu)$, rapprochée du pharynx et souvent recouverte par l'organe tribocytique arrondi ou largement elliptique (530-950/570-1020 $\mu$ ), s'ouvrant par une fente médiane. L'ovaire est réniforme, intersegmentaire (150-190/340-420 $\mu$ ). Les œufs, nombreux ou très nombreux, mesurent 105-130/65-78 $\mu$.

Fibricola lucida (La Rue et Bosma, 1927)

Ce parasite de l'Opossum a été découvert au Texas (LA RuE et Bosma, 1927; Read, 1948), puis signalé en Louisiane (DikMans, 1931; LUMSDEN et ZischKe, 
1961), dans l'Oklahoma (PARK, 1936) et dans l'Illinois (BABERO, 1957), au Tennessee et en Georgie (Byrd, Reiber et PARKer, 1942). ReAd (op. cit.) puis Lumsden et Zischke (op. cit.) l'ont aussi trouvé chez le Vison (Mustela vison Schr.), au Texas et en Louisiane.

M. John M. Kinsella, de l'Université de Floride, à Gainesville, nous a remis deux lots de Fibricola lucida provenant du duodénum du «Rice Rat», Oryzomys palustris (Harlan) [Cricetidae], l'un recueilli à Cedar Key, Levy County, Fla., le 12 juin 1970, l'autre récolté à Paynes Prairie, Alachua County, Fla., le 21 juillet 1970 (extensité: plus de $50 \%$ pour ce second lot).

Les follicules vitellogènes erratiques du second segment sont nettement visibles; ils atteignent, en deux bandes, le niveau de la vésicule séminale ou même celui du rebord antérieur de la bourse copulatrice.

\section{Braunina cordiformis Wolf, 1903}

$M^{1 l e}$ E. van den Broek a eu l'amabilité de nous envoyer 3 exemplaires de ce Ver, fixés à des fragments de la muqueuse d'un Tursiops truncatus (Montagu). Ce Dauphin souffleur fut trappé à Grassy Key (Florida, U.S.A.) et transporté par le $D^{r}$ W. H. Dudok van Heel au « Dolphinarium » de Harderwijk (Pays-Bas), où il mourut le 25 juin 1968.

Braunina cordiformis a été décrit par HeIDER (1900, pp. 19-22) sans désignation spécifique, d'après des exemplaires provenant d'une part de l'intestin de deux Dauphins, - l'un disséqué à la Station zoologique de Trieste, en 1877, l'autre capturé à Lesina par le professeur C. Heller et dont les parasites furent déposés au Musée zoologique d'Innsbruck, - d'auțre part de l'intestin d'un «Delphin » hébergeant des Vers récoltés par Natterer à Rio de Janeiro et conservés au Musée d'Histoire naturelle de Vienne (n ${ }^{\circ}$ 4689) (E. van den Broek, in litteris, 18 novembre 1968). ${ }^{1}$

Ce parasite de Delphinidés, dont l'étude morphologique, anatomique et histologique a été faite par Wolf (1903), fut retrouvé par MCINTOSH (1953) en Floride, chez Tursiops truncatus (Montagu), attaché aux parois des "premier et second estomacs », puis dans la baie de Panama, chez Stenella graffmani Lönnberg. Hutton et Sogandares-Bernal (1960), puis Hutton (1964) le signalent en Floride (St. Petersburg Beach), chez Tursiops truncatus. Le second auteur nous a remis quelques spécimens prélevés dans l'estomac du Dauphin souffleur, aussi en Floride (Boca Ciega Bay, coll. F. Sogandares). Enfin, Schryver, Medway et Williams (1967) retrouvent le Ver dans les estomacs fundique et pylorique de trois Tursiops truncatus provenant d'« Aquarama », Philadelphia, Pa., et d'«Aquatics International », Berlin, N. J.

${ }^{1}$ L'indication «Darm von Squalus, Rio de J.» doit résulter d'une confusion. 


\section{BIBLIOGRAPHIE}

Babero, B. B. 1957. Some helminths from Illinois opossums. J. Parasit. 43: 232.

Byrd, E. E., R. J. Reiber et M. V. PARKer. 1942. Mammalian trematodes. I. Trematodes from the opossum, Didelphis virginiana Kerr. J. Tenn. Acad. Sci. 17: 130-142.

Dikmans, G. 1931. A new nematode worm, Viannaia bursobscura, from the opossum, with a note on other parasites of the opossum. Proc. U. S. natn. Mus. 79: $1-4$.

Dubors, G. 1938. Monographie des Strigeida (Trematoda). Mém. Soc. neuchât. Sci. nat. $6: 1-535$.

DuboIs, G. 1967. Notes helminthologiques. I: Strigeidae Railliet (Trematoda). Revue suisse Zool. 74: 693-700.

Dubois, G. 1968. Synopsis des Strigeidae et des Diplostomatidae (Trematoda). Mém. Soc. neuchât. Sci. nat. 10: 1-258.

DuboIs, G. 1969. Notes helminthologiques. II: Diplostomatidae Poirier et Cyathocotylidae Poche (Trematoda). Revue suisse Zool. 76: 3-21.

Dubois, G. et R. RausCh. 1950. Troisième contribution à l'étude des Strigeides (Trematoda) nord-américains. Bull. Soc. neuchât. Sci. nat. 73: 19-50.

DuboIs, G. et R. RausCH. 1960. Quatrième contribution à l'étude des Strigeides (Trematoda) nord-américains. Ibid. 83: 79-92.

Dubois, G. et R. L. RausCH. 1964. Studies on the helminth fauna of Alaska. XL. Strigea gruis sp. n., a trematode parasite of Grus canadensis (L.). J. Parasit. 50: $445-447$.

DYER, W. G. 1970. Helminths of the striped skunk, Mephitis mephitis Schreber, in North Dakota. Proc. helminth. Soc. Wash. 37: 92-93.

Hutton, R. F. 1964. A second list of parasites from marine and coastal animals of Florida. Trans. Am. microsc. Soc. 83: 439-447.

Hutton, R. F. et F. Sogandares-Bernal. 1960. A list of parasites from marine and coastal animals of Florida. Trans. Am. microsc. Soc. 79: 287-292.

La Rue, G. R. et N. J. Bosma. 1927. Studies on the trematode family Strigeidae (Holostomidae). Neodiplostomum lucidum n. sp. J. Parasit. 14: 124-125.

Lumsden, R. D. et J. A. ZischKe. 1961. Seven trematodes from small mammals in Louisiana. Tulane Stud. Zool. 9: 87-98.

McInTosh, A. 1953. New hosts and distribution records for the trematode genus Braunina Heider, 1900. J. Parasit. 39: 31 (Abstract).

NiewiadomsKa, K. 1970. An analysis of criteria for generic differenciation within the order Strigeidida (La Rue, 1926). Acta parasit. pol. 18: 277-289.

Odening, K. 1970. Neue Funde von Diplostomidae (Trematoda) aus Vögeln des Berliner Tierparks. Mitt. zool. Mus. Berl. 46: 167-181.

PARK, P. J. 1936. The miracidium of Neodiplostomum lucidum La Rue and Bosma. Trans. Am. microsc. Soc. 55: 49-54.

Pearson, J. C. 1959. Observations on the morphology and life cycle of Strigea elegans Chandler and Rausch, 1947 (Trematoda: Strigeidae). J. Parasit. 45: $155-174$.

READ, C. P. 1948. Strigeids from Texas mink with notes on the genus Fibricola Dubois. Trans. Am. microsc. Soc. 67: 165-168. 
Schryver, H. F., W. Medway et J. F. Williams. 1967. The stomach fluke Braunina cordiformis in the Atlantic bottlenose dolphin. J. Am. vet. med. Ass. 151: 884-886.

Sitko, J. 1968. Trematodes of birds of the family Laridae in Czechoslovakia. Věst. čsl. Spol. zool. 32: 275-292.

SitKo, J. 1969. Findings of trematodes (Trematoda) in wild birds of Czechoslovakia. Věst. čsl. Spol. zool. 33: 79-87.

Ukoli, F. M. A. 1967. On Apharyngostrigea (Apharyngostrigea) simplex (Johnston, 1904) new comb. and $A$. (Apharyngostrigea) serpentia n. sp. (Strigeidae: Trematoda) with an evaluation of the taxonomy of the genus Apharyngostrigea Ciurea, 1927, by the method of numerical taxonomy. $\mathrm{J}$. $\mathrm{Hel}$ minth. 41: 235-256.

Wolf, K. 1903. Beitrag zur Kenntnis der Gattung Braunina Heider. Sber. Akad. Wiss. Wien 112: 603-626.

Adresse de l'Auteur:

G. Dubois,

Grand-Rue 12,

CH-2035 Corcelles. 


\section{$2 \mathrm{BHL}$ Biodiversity Heritage Library}

Dubois, Georges. 1974. "Notes Helminthologiques III: Strigeidae Railliet, Diplostomidae Poirier et Brauninidae Bosma (Trematoda)." Revue suisse de zoologie 81, 29-39. https://doi.org/10.5962/bhl.part.75985.

View This Item Online: https://www.biodiversitylibrary.org/item/126812

DOI: https://doi.org/10.5962/bhl.part.75985

Permalink: https://www.biodiversitylibrary.org/partpdf/75985

\section{Holding Institution}

Smithsonian Libraries

\section{Sponsored by}

Biodiversity Heritage Library

\section{Copyright \& Reuse}

Copyright Status: In Copyright. Digitized with the permission of the rights holder.

Rights Holder: Muséum d'histoire naturelle - Ville de Genève License: http://creativecommons.org/licenses/by-nc-sa/3.0/

Rights: https://www.biodiversitylibrary.org/permissions/

This document was created from content at the Biodiversity Heritage Library, the world's largest open access digital library for biodiversity literature and archives. Visit BHL at https://www.biodiversitylibrary.org. 